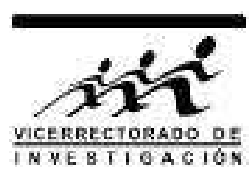

\title{
Dependencia en energía de la producción de picos de escape en espectroscopia de fluorescencia de rayos- $X$ dispersiva en energía
}

\author{
Jorge A. Bravo Cabrejos* \\ Facultad de Ciencias Físicas, Universidad Nacional Mayor de San Marcos, Lima, Perú
}

Recibido 21 mayo 2015 - Aceptado 20 julio 2015

\begin{abstract}
Se presenta el modelo teórico para calcular la energía e intensidad relativa de los picos de escape correspondientes a los rayos- $X$ característicos presentes en un espectro de fluorescencia de rayos- $X$ dispersiva en energía (FRXDE). Los picos de escape de elementos presentes en una muestra en alta concentración interfieren con la identificación de rayos-X característicos de elementos presentes en muy baja concentración. El resultado de este análisis se utiliza para simular con buenos resultados la presencia de los picos de escape de una muestra de ceniza de madera que contiene $\mathrm{K}$ y $\mathrm{Ca}$ en alta concentración

Palabras claves: Picos de escape, fluorescencia de rayos- $X$ dispersiva en energía, ceniza de madera.
\end{abstract}

\section{Energy dependence of the production of escape peaks in energy dispersive $\mathrm{X}$-ray fluorescence spectroscopy}

A theoretical model is presented to calculate the energy and relative intensity of escape peaks corresponding to the characteristic $X$-rays present in an energy dispersive $\mathrm{X}$-ray fluorescence (EDXRF) spectrum. The escape peaks of elements present in high concentration in a sample interfere with the identification of characteristic X-rays of elements present in low concentration. The results of this analysis have been used to simulate with good results the presence of the escape peaks in a sample of wood ashes with high concentration of $\mathrm{K}$ and $\mathrm{Ca}$.

Keywords: escape peals, energy dispersive $\mathrm{X}$-ray fluorescence, wood ashes.

Recientemente la Facultad de Ciencias Físicas ha adquirido de la empresa AMPTEK un nuevo equipo portátil de fluorescencia de rayos- $X$ dispersiva en energía. La cadena de detección de rayos- $X$ de baja energía de este equipo posee una mejor resolución en energía, 140 $\mathrm{eV}$ a mitad de altura comparado al anterior de $200 \mathrm{eV}$ para rayos-X de $6.40 \mathrm{keV}$. Esta mejor resolución permite resolver mejor a simple vista la presencia de picos de rayos-X característicos de energías cercanas; también permite resolver la presencia de los débiles picos de escape, cuya presencia debe tomarse en cuenta en el análisis cualitativo y sobre todo en el análisis cuantitativo de los espectros. En estas circunstancias se ha visto perentorio realizar un estudio detallado de la producción de los picos de escape a fin de que sea incluído en el programa de simulación de espectros de FRXDE que utiliza el Laboratorio de Arqueometría.

El detector del equipo de FRXDE utiliza un cristal de Si-PIN de $500 \mu \mathrm{m}$ de espesor y $3 \mathrm{~mm}$ de diámetro. Los picos de escape se producen cuando los rayos- $X$ característicos que emiten estos átomos de $\mathrm{Si}$ logran salir del cristal sin liberar energía dentro del cristal; al

*jbravoc@unmsm.edu.pe 
darse este fenómeno, el sistema de detección produce un pulso que se asocia a un rayo- $X$ con energía igual a la energía del rayos- $X$ incidente menos la energía del rayo-X característico de Si que abandona el sistema de detección.

Por tener mayor energía y, por lo tanto, un mayor recorrido libre en el $\mathrm{Si}$, en este estudio tomamos en cuenta solamente los rayos- $X \mathrm{~K}_{\alpha}$ y $\mathrm{K}_{\beta}$ de este elemento. Para nuestros cálculos usamos las propiedades físicas del $\mathrm{Si}$, tales como la densidad $\rho=2.33 \mathrm{~g} / \mathrm{cm}^{3}$; el número atómico $Z=14$; la estructura electrónica $1 s^{2} 2 S^{2} 2 p^{6} 3 s^{2} 3 p^{2}$; el potencial de ionización de la capa $\mathrm{K}, I_{K}=1.838 \mathrm{keV}$; la probabilidad de arrancar un electrón $\mathrm{K}$ cuando el átomo interactúa con un fotón de rayos-X con energía $h \nu>I_{K}, F_{K}=0.9125$; la probabilidad de liberar energía vía emisión de los rayos- $\mathrm{X} \mathrm{K}$ luego de crearse una vacancia en la capa $\mathrm{K}, \omega K=0.0495$ [1]; la probabilidad de emisión de un rayo- $\mathrm{X} \mathrm{K}_{\alpha}, P_{\alpha}=0.9576$; la probabilidad de emisión de un rayo- $\mathrm{X} \mathrm{K} \mathrm{K}_{\beta}, P_{\beta}=0.0424$.

\begin{tabular}{llll}
\hline $\begin{array}{l}\text { Energía } \\
(\mathrm{keV})\end{array}$ & $\begin{array}{l}\text { Efecto Fo- } \\
\text { toeléctrico } \\
\left(\mathrm{cm}^{2} / \mathrm{g}\right)\end{array}$ & $\begin{array}{l}\text { Total } \\
\left(\mathrm{cm}^{2} / \mathrm{g}\right)\end{array}$ & $\begin{array}{l}\text { Recorrido } \\
\text { libre medio } \\
(\mu \mathrm{m})\end{array}$ \\
\hline 1 & 1570 & 1572 & 2.73 \\
1.2 & 965 & 968 & 4.43 \\
1.4 & 641 & 643 & 6.67 \\
1.6 & 448 & 450 & 9.54 \\
$1.739\left(\mathrm{~K}_{\alpha}\right)$ & 367 & 369 & 11.9 \\
$1.829\left(\mathrm{~K}_{\beta}\right)$ & 311 & 313 & 13.7 \\
$1.837-$ & 307 & 309 & 13.9 \\
$1.837+$ & 3191 & 3193 & 1.33 \\
2 & 2770 & 2780 & 1.54 \\
4 & 451 & 453 & 9.47 \\
6 & 146 & 147 & 29.2 \\
8 & 64 & 65 & 66.3 \\
10 & 33 & 34 & 127 \\
12 & 19 & 20 & 227 \\
\hline
\end{tabular}

Tabla 1: Coeficientes de atenuación másicos para efecto fotoeléctrico y total de silicio. Se incluye el recorrido medio libre para las energías citadas.

La Tabla 1 muestra el valor de la sección eficaz másica total y para efecto fotoeléctrico de Si para energías en el intervalo de $1 \mathrm{keV}$ a $12 \mathrm{keV}$. Este intervalo incluye el borde de ionización de la capa $\mathrm{K}$ que yace a una energía de $1.829 \mathrm{keV}$. Estos valores de las propiedades atómicas de $\mathrm{Si}$ y de sus secciones eficaces se han obtenido accediendo a los portales del OIEA [2] y del NIST [3] y han servido de base para los cálculos realizados. Esta Tabla también lista el recorrido libre medio, $\lambda$, calculado de rayos-X para las energías mencionadas, $\mathrm{y}$ que viene dado por

$$
\lambda=\frac{1}{\rho \sigma_{t}},
$$

donde $\sigma_{t}$ es la sección transversal total másica, que depende de la energía de los rayos- $X$. Para los rayos- $X K$ de $\mathrm{Si}, \lambda$ es del orden de $12 \mu \mathrm{m}$, lo cual indica que los rayos- $\mathrm{X} \mathrm{K}$ que logran escapar son producidos en una capa superficial de unos $30 \mu \mathrm{m}$ de espesor.

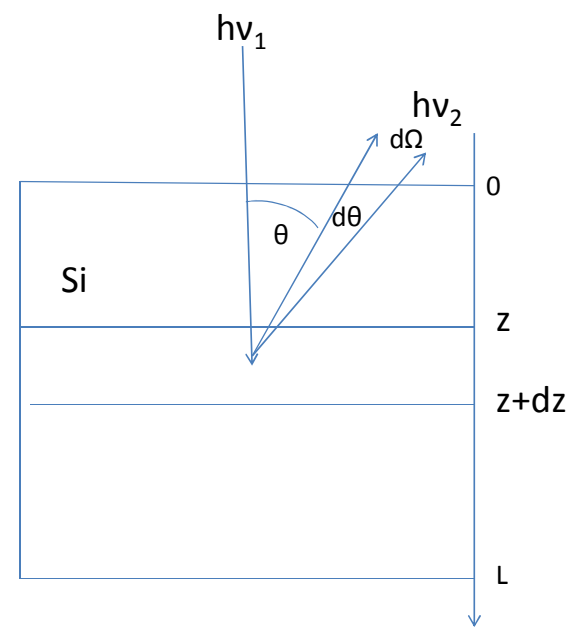

Figura 1: Vista transversal de la geometría utilizada para el análisis de los fotones atravesando el detector.

A continuación se calcula la probabilidad de escape promedio de un rayos- $\mathrm{X} \mathrm{K}$ de $\mathrm{Si}$ que es producido por un rayo- $X$ que incide de manera perpendicular a la superficie de Si del detector y lejos del borde del cristal. La Figura 1 muestra el arreglo geométrico correspondiente. Esta probabilidad está dado por

$$
P_{E}=\int_{0}^{L} \sigma_{f_{1}} d z \int_{0}^{\pi / 2} \exp \left[-\sigma_{t_{1}} z-\frac{\sigma_{t_{2}} z}{\cos \theta}\right] \frac{d \Omega}{4 \pi}
$$

donde $\sigma_{f_{1}}, \sigma_{t_{1}}$ y $\sigma_{t_{2}}$ son las secciones eficaces lineales para efecto fotoeléctrico de Si con el rayo-X incidente, $y$ totales de $\mathrm{Si}$ con el rayo-X incidente y el rayo-X emitido, respectivamente; $L$ es el espesor del detector de $\mathrm{Si}$, que se puede considerar como infinito; $d \Omega$ es el elemento de ángulo sólido hacia el cual es emitido el rayo- $\mathrm{X} \mathrm{K}$ de Si. La función $\exp \left[-\sigma_{t_{1}} z\right] \sigma_{f_{2}} d z$ es la fracción de los rayos- $X$ incidentes que interactúan con el detector vía el efecto fotoeléctrico en el intervalo $(z, z+d z)$, mientras que, la función $\exp \left[-\sigma_{t_{2}} z / \cos \theta\right] d \Omega / 4 \pi$ es la fracción de los rayos- $X$ de $\mathrm{Si}$ producidos que son emitidos hacia el $d \Omega$ de manera aleatoria y logran atravesar el detector. 
Luego, se aplica el cambio de variable $\mu=\cos \theta$, con $-1 \leq \mu \leq 1, d \Omega=2 \pi \operatorname{sen} \theta d \theta=2 \pi d \mu$, y se ejecuta la integración sobre la variable $z$, considerando que $0 \leq \mu \leq 1$ es el intervalo de interés. Finalmente, la probabilidad de escape es

$$
\begin{aligned}
P_{E} & =\frac{1}{2} \int_{0}^{1} \frac{\sigma_{f_{1}}}{\sigma_{t_{1}}+\frac{\sigma_{t_{2}}}{\mu}} \\
& =\frac{\sigma_{f_{1}}}{2 \sigma_{t_{1}}}\left[1-\frac{\sigma_{t_{2}}}{\sigma_{t_{1}}} \ln \left(1+\frac{\sigma_{t_{1}}}{\sigma_{t_{2}}}\right)\right],
\end{aligned}
$$

como una función que depende de la energía de los rayos- $\mathrm{X}$ incidentes y de la energía de los rayos- $\mathrm{X}$ de $\mathrm{Si}$ considerando $\mathrm{K}_{\alpha} \circ \mathrm{K}_{\beta}$.

Para calcular la probabilidad que se produzca un pico de escape por cada rayo- $X$ incidente, hay que tomar en cuenta además la probabilidad $F_{K}$ que se crea una vacancia en la capa $\mathrm{K}$ de $\mathrm{Si}$ y el rendimiento de emisión de un rayo- $\mathrm{X} \mathrm{K}, \omega K$. El efecto Auger compite de manera favorable en este caso. Por lo tanto, la probabilidad que se produzca un pico de escape por cada rayo-X in- cidente de cierta energía $E_{\alpha}=h \nu_{1}$ (en keV) viene dada por

$$
\begin{aligned}
P_{e \alpha}= & P_{\alpha} \omega K F_{K} P_{E}\left(h \nu_{1}, h \nu_{2}\right) \\
= & 0.0320-0.00965 E_{x} \\
& +0.00106 E_{x}^{2}-4.1 \times 10^{-5} E_{x}^{3}
\end{aligned}
$$

para un rayo- $\mathrm{X} \mathrm{K}_{\alpha} \mathrm{y}$

$$
\begin{aligned}
P_{e \beta}= & P_{\beta} \omega K F_{K} P_{E}\left(h \nu_{1}, h \nu_{2}\right) \\
= & 0.00142-4.1 \times 10^{-3} E_{x} \\
& +4.4 \times 10^{-5} E_{x}^{2}-1.7 \times 10^{-6} E_{x}^{3}
\end{aligned}
$$

para un rayo- $\mathrm{X} \mathrm{K}_{\beta}$.

La Tabla 2 muestra las energías donde aparecen los picos de escape para cada rayo- $\mathrm{X} \mathrm{K}$ de los elementos con $17 \leq Z \leq 30$ y el número de rayos $\mathrm{K}_{\alpha}$ que escapan por cada mil fotones de rayos- $X$ de cada tipo que interactúan con el detector. Como se puede observar este número varia de $13.1 / 1000$ para $\mathrm{Cl} \mathrm{K}_{\alpha}$ a 1.1/1000 para $\mathrm{Zn} \mathrm{K}$.

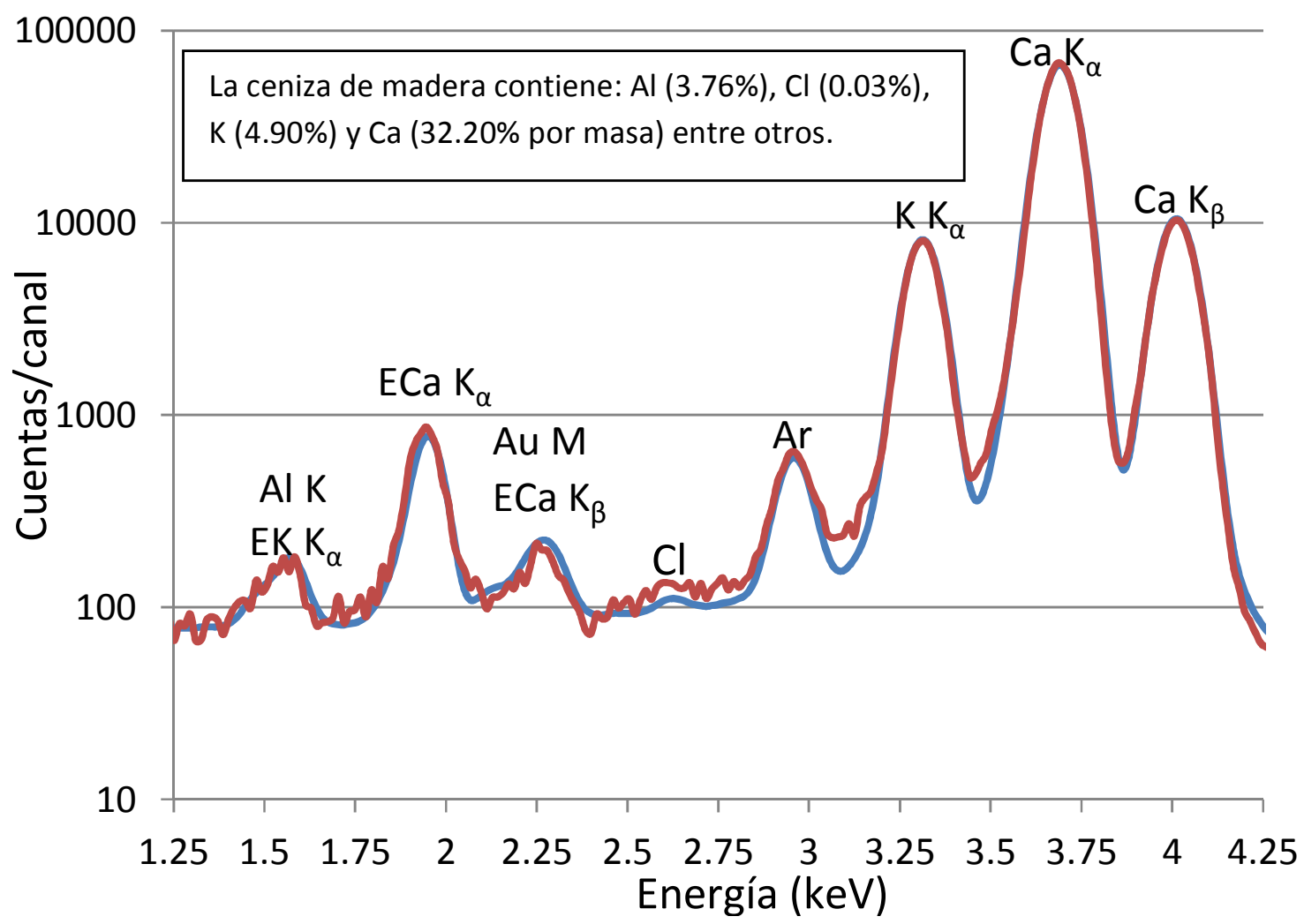

Figura 2: Espectro de FRXDE de ceniza de madera mostrando los picos de los rayos-X K de Al, Ar, $\mathrm{K}$ y Ca y los respectivos picos de escape. Se muestran los datos experimentales (en rojo) y el espectro calculado (en azul). 


\begin{tabular}{|c|c|c|c|c|c|}
\hline Elemento & $\begin{array}{ll}\begin{array}{l}\text { Energía } \\
\text { rayo-X }\end{array} & \mathrm{K} \\
(\mathrm{keV}) & \\
\end{array}$ & $\begin{array}{l}\text { Coef. de } \\
\text { atenuación } \\
\left(\mathrm{cm}^{2} / \mathrm{g}\right)\end{array}$ & $\begin{array}{ll}\begin{array}{l}\text { Energía del } \\
\text { pico }\end{array} & \mathrm{K}_{\alpha} \\
(\mathrm{keV}) & \\
\end{array}$ & $\begin{array}{ll}\begin{array}{l}\text { Energía del } \\
\text { pico }\end{array} & \mathrm{K}_{\beta} \\
(\mathrm{keV}) & \\
\end{array}$ & $\begin{array}{l}\text { № } \mathrm{K}_{\alpha} \text { que } \\
\text { escapan/ } \\
10^{3}\end{array}$ \\
\hline \multirow[t]{2}{*}{$\mathrm{Cl}$} & 2.621 & 1440 & 0.882 & 0.792 & 13.1 \\
\hline & 2.815 & 1199 & 1.076 & 0.986 & 12.2 \\
\hline \multirow[t]{2}{*}{$\mathrm{Ar}$} & 2.957 & 1054 & 1.218 & 1.128 & 11.6 \\
\hline & 3.190 & 860 & 1.451 & 1.361 & 10.7 \\
\hline \multirow[t]{2}{*}{ K } & 3.312 & 777 & 1.573 & 1.483 & 10.2 \\
\hline & 3.509 & 622 & 1.850 & 1.760 & 9.2 \\
\hline \multirow[t]{2}{*}{$\mathrm{Ca}$} & 3.690 & 575 & 1.951 & 1.861 & 8.8 \\
\hline & 4.012 & 454 & 2.273 & 2.183 & 7.7 \\
\hline \multirow[t]{2}{*}{$\mathrm{Sc}$} & 4.068 & 436 & 2.329 & 2.239 & 4.5 \\
\hline & 4.480 & 331 & 2.741 & 2.651 & 6.4 \\
\hline \multirow[t]{2}{*}{$\mathrm{Ti}$} & 4.506 & 326 & 3.001 & 2.911 & 6.3 \\
\hline & 4.931 & 252 & 3.426 & 3.336 & 5.3 \\
\hline \multirow[t]{2}{*}{ V } & 4.949 & 249 & 3.210 & 3.120 & 5.2 \\
\hline & 5.426 & 102 & 3.681 & 3.591 & 4.3 \\
\hline \multirow[t]{2}{*}{$\mathrm{Cr}$} & 5.411 & 194 & 3.672 & 3.582 & 4.4 \\
\hline & 5.924 & 150 & 4.185 & 4.095 & 3.6 \\
\hline \multirow[t]{2}{*}{$\mathrm{Mn}$} & 5.894 & 152 & 4.155 & 4.065 & 3.5 \\
\hline & 6.489 & 117 & 4.750 & 4.660 & 2.8 \\
\hline \multirow[t]{2}{*}{$\mathrm{Fe}$} & 6.398 & 122 & 4.660 & 4.570 & 3.0 \\
\hline & 7.057 & 94 & 5.318 & 5.228 & 2.4 \\
\hline \multirow[t]{2}{*}{ Co } & 6.924 & 98 & 5.185 & 5.095 & 2.5 \\
\hline & 7.648 & 76 & 5.909 & 5.819 & 2.0 \\
\hline \multirow[t]{2}{*}{$\mathrm{Ni}$} & 7.471 & 80 & 5.732 & 5.642 & 2.1 \\
\hline & 8.263 & 61 & 6.529 & 6.439 & 1.7 \\
\hline \multirow[t]{2}{*}{$\mathrm{Cu}$} & 8.040 & 66 & 6.301 & 6.211 & 1.8 \\
\hline & 8.904 & 50 & 7.165 & 7.075 & 1.4 \\
\hline \multirow[t]{2}{*}{ Zn } & 8.630 & 54 & 6.891 & 6.801 & 1.5 \\
\hline & 9.570 & 40 & 7.831 & 7.751 & 1.1 \\
\hline
\end{tabular}

Tabla 2: Energías de los rayos- $\mathrm{X} \mathrm{K}$ y de sus respectivos picos de escape para los elementos de interés, incluyendo los coeficientes de atenuación y el número de rayos- $X$ de escape que se producen por cada mil que interactúan con el detector.

Para poner a prueba estos resultados se considera el espectro de FRXDE de una muestra de ceniza de madera que contiene: $\mathrm{Al}(3.76 \%), \mathrm{Cl}(0.03 \%), \mathrm{K}(4.90 \%)$ y $\mathrm{Ca}(32.20 \%$ por masa) entre otros elementos. La Figura 2 muestra el espectro a baja energía donde se puede apreciar los picos de escape de $\mathrm{K}$ y $\mathrm{Ca}$. Las amplitudes de estos picos de escape se han calculado de acuerdo a la teoría presentada, la cual ha sido incorporada al programa de simulación de espectros de FRXDE. Como se puede observar la presencia de estos picos de escape puede interferir en el análisis de la presencia de $\mathrm{Al}, \mathrm{Si}, \mathrm{P}$ y $\mathrm{S}$; además, el pico de escape de $\mathrm{Ca}_{\beta}$ interfiere con los rayos- $X \mathrm{M}$ de $A u$ que emite la fuente de rayos- $X$.

En conclusión, los resultados del análisis presentado de los picos de escape se han aplicado al espectro de ceniza de madera de manera satisfactoria; ha permitido reproducir los datos experimentales correspondientes a los picos de escape de $\mathrm{K}$ y Ca. De manera particular, este ejemplo muestra cómo la presencia del pico de escape de $\mathrm{K}$ interfiere con la presencia de los rayos- $\mathrm{X} \mathrm{K}$ de $\mathrm{Al}$; de ignorarse su presencia conllevaría a exagerar la concentración de $\mathrm{Al}$ en la muestra.

Se agradece el apoyo económico recibido del Consejo Superior de Investigaciones del Vicerrectorado de la UNMSM a través del proyecto con código 1513011071. Asimismo, se agradece las facilidades otorgadas por el Laboratorio de Arqueometría de la Facultad. 


\section{Referencias}

[1] T. L. Hopman, An accurate determination of $K$ shell $X$-ray fluorescece yield of silicon, X-ray Spectrometry 41(21), 164 (2012).
[2] OIEA, Quantitative X-ray Analysis System, Agencia Internacional de Energía Atómica, Viena (1995).

[3] M. J. Berger, XCOM: Photon Cross Sections Database, National Institute of Standard and Technology, Washington (1998). 